\title{
Brauchen wir EMH? Was braucht EMH?
}

\section{Ruedi Bienz}

zum Abschied als Geschäftsführer von EMH

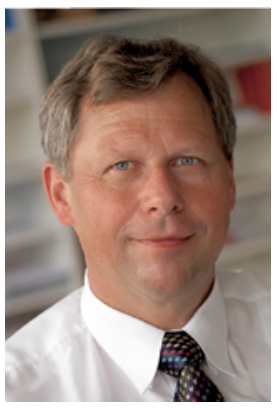

Bei einer Verabschiedung hört man oft von «einem Kind, das man in gute Hände übergibt» oder von «Herzblut, mit dem man während Jahren ...». Ich möchte hier etwas nüchterner bleiben und kurz zurück, aber vorwiegend in die Zukunft schauen.

Im Jahre 1997 suchte eine Arbeitsgruppe der FMH mit dem damaligen Präsidenten Hans Heinrich Brunner, dem Zentralvorstandsmitglied Ludwig Heuss und dem Chefredaktor der SÄZ, Reto Steiner, das Gespräch mit verschiedenen Schweizer Verlagen. Anvisiert wurde die Zusammenarbeit mit einem Verlag, der das medizinische und verlegerische Fachwissen mitbrachte, um gemeinsam mit der FMH folgende Ziele zu erreichen:

- die medizinische Publizistik in der Schweiz durch redaktionell unabhängige, qualitativ hochwertige Publikationen zu prägen;

- die FMH und den Zusammenhalt unter ihren Mitgliedern zu stärken;

- die Wahrnehmung der Ärzteschaft in der Öffentlichkeit positiv zu prägen.

Das Evaluationsverfahren resultierte in der Zusammenarbeit mit dem Wissenschaftsverlag Schwabe, dessen Konzept überzeugte und der eigene Zeitschriften und Medizinwerke in den gemeinsamen Verlag einbringen konnte. Am 18. September 1997 wurde der Schweizerische Ärzteverlag EMH gegründet, der - ein nicht unwesentliches Detail - auch Gewinn für die beteiligten Partner abwerfen sollte.

Bald zeigte sich, dass die hochgesteckten Ziele gemessen an der Realität der medizinischen Publizistik in der Schweiz als sehr idealistisch einzustufen waren. Der Kern des Problems ist bis heute derselbe geblieben die grosse Stärke der EMH-Produkte ist zugleich ihre Achillesferse. Denn sämtliche von EMH herausgegebenen Zeitschriften basieren auf absoluter redaktioneller Unabhängigkeit und orientieren sich ausschliesslich an den Bedürfnissen der Leserschaft. Auf dieser Kompromisslosigkeit basiert das Vertrauen, das die Leserschaft unseren Produkten entgegenbringt. In unabhängigen Leserumfragen $[1,2]$ belegen EMH-Zeitschriften stets die Spitzenpositionen - mit grossem Abstand auf die Konkurrenz. Für die Gewinnung von Werbegeldern der Industrie ist das Konzept der strikten Trennung von Redaktion und Marketing eher ein Störfaktor, da Inserate von den Werbekunden primär nach anderen Kriterien platziert werden.

Die sich aus dieser zunächst unterschätzten Konstellation ergebenden finanziellen Schwierigkeiten führten um die Jahrtausendwende zu meiner Berufung als Geschäftsführer von EMH durch die beiden Aktionäre FMH und Schwabe. Mit Unterstützung der Aktionäre und dank eines motivierten Teams gelang es, EMH auf die Erfolgsspur zu bringen. Auch 21 Jahre nach der Gründung gibt es EMH noch - und das Unternehmen ist wirtschaftlich gesund. Die Herausforderungen, mit denen EMH in der sich rasend schnell verändernden Medienwelt konfrontiert ist, sind allerdings beträchtlich.

Als scheidender Geschäftsführer darf ich frei von der Leber sprechen beziehungsweise schreiben. Um das Modell EMH erfolgreich weiterzuführen, muss die Ärzteschaft, müssen Sie, liebe Leserinnen und Leser, hinter dem oben skizzierten Auftrag an EMH stehen. Es ist aus meiner Sicht zentral, dass Sie sich der Tragweite dieses Auftrages, der Relevanz eines eigenen Verlages, und der damit verbundenen Verantwortung bewusst sind und diese Verantwortung mittragen. Sie erhalten von EMH (nahezu) unentgeltlich [3] jede Woche die Schweizerische Ärztezeitung und das Swiss Medical Forum zugestellt. EMH stellt Ihnen mit dem Swiss Medical Weekly zudem eine wissenschaftliche Publikationsplattform mit einem ansehnlichen Impact Factor zur Verfügung. Und schliesslich bietet EMH Fachgesellschaften eine professionelle Infrastruktur, mit der sie ihre eigene Kommunikation zeitgemäss und kostengünstig führen können. Die Finanzierung dieser Dienstleistungen erfolgt nicht über ihren Mitgliederbeitrag, sondern durch mühsam verdiente Werbegelder.

Um in einem hochkompetitiven und sich rasch verändernden medialen Umfeld bestehen zu können, braucht EMH kurze Entscheidungswege, Flexibilität und unternehmerische Freiheit. Deshalb zum Schluss der Wunsch und die Bitte an Sie, geschätzte Leserinnen und Leser, geschätzte Mitglieder der FMH: Geben Sie EMH via die zuständigen Gremien der FMH die notwendigen Freiheiten, schenken Sie dem Verwaltungsrat Vertrauen und lassen Sie EMH gute Arbeit machen. 